\title{
Algorithms for Piecewise Polynomials and Splines with Free Knots
}

\author{
By G. Meinardus, G. Nürnberger, M. Sommer, and H. Strauss
}

\begin{abstract}
We describe an algorithm for computing points $a=x_{0}<x_{1}<\cdots<$ $x_{k}<x_{k+1}=b$ which solve certain nonlinear systems $d\left(x_{i-1}, x_{i}\right)=d\left(x_{i}, x_{i+1}\right), i=$ $1, \ldots, k$. In contrast to Newton-type methods, the algorithm converges when starting with arbitrary points. The method is applied to compute best piecewise polynomial approximations with free knots. The advantage is that in the starting phase only simple expressions have to be evaluated instead of computing best polynomial approximations. We finally discuss the relation to the computation of good spline approximations with free knots.
\end{abstract}

0. Introduction. Let $[a, b]$ be an interval of the real line and $k$ be a natural number. Moreover, let $D=\left\{(x, y) \in \mathbf{R}^{2}: a \leq x \leq y \leq b\right\}$ and $d: D \rightarrow \mathbf{R}$ be a function with the following properties:

$$
\begin{aligned}
& d \text { is continuous, } \\
& d(x, x)=0 \text { for all }(x, x) \in D, \\
& d(x, y) \leq d(\tilde{x}, \tilde{y}) \quad \text { if }[x, y] \subset[\tilde{x}, \tilde{y}] \subset[a, b] .
\end{aligned}
$$

A partition $a=x_{0} \leq x_{1} \leq \cdots \leq x_{k} \leq x_{k+1}=b$ is called a leveled set if

$$
d\left(x_{i-1}, x_{i}\right)=d\left(x_{i}, x_{i+1}\right), \quad i=1, \ldots, k,
$$

and it is called an optimal set if

$$
\max _{0 \leq i \leq k} d\left(x_{i}, x_{i+1}\right) \leq \max _{0 \leq i \leq k} d\left(y_{i}, y_{i+1}\right)
$$

for all knots $a=y_{0} \leq y_{1} \leq \cdots \leq y_{k} \leq y_{k+1}=b$. It is easy to verify that every leveled set is optimal. Using the idea of an algorithm for segment approximation in [10], in Section 1 we give an algorithm to compute a sequence of knot sets converging to a leveled (and therefore to an optimal) set. Simultaneously, a sequence converging to the optimal value

$$
m_{k}=\min _{\left\{y_{1}, \ldots, y_{k}\right\}} \max _{0 \leq i \leq k} d\left(y_{i}, y_{i+1}\right)
$$

is determined. In contrast to Newton-type methods, the algorithm converges for arbitrarily chosen (e.g., equidistant) knots.

In Section 2 we apply the above algorithm to best uniform approximation of a given function $f \in C[a, b]$ by piecewise polynomials with free knots. This approximation problem will be solved in two phases.

Received November 20, 1987.

1980 Mathematics Subject Classification (1985 Revision). Primary 41A15, 41A10, 65D07; Secondary $41 \mathrm{~A} 50,65 \mathrm{D} 15,65 \mathrm{H} 10$. 
In phase one we choose a suitable function $d$ which is derived from results on the minimal deviation in best polynomial approximation and compute a leveled set of knots for $d$. Starting with this set of knots, in phase two we apply our algorithm developed in [10] to the function

$$
d(x, y)=\min _{p \in \Pi_{m}}\|f-p\|_{[x, y]}
$$

where $\Pi_{m}$ is the space of polynomials of degree at most $m$ and $\|\cdot\|$ denotes the supremum norm. We obtain an optimal set of knots for best piecewise polynomial approximation of $f$.

The advantage of the two-phase method is that in phase one, only a simple expression $d\left(x_{i}, x_{i+1}\right)$ has to be evaluated, while in phase two we have to apply the Remez algorithm to compute the value $d\left(x_{i}, x_{i+1}\right)$. Moreover, our numerical results show that in most cases the set of knots obtained in phase one is already nearly optimal.

We finally use these optimal knots as fixed knots and compute a corresponding best spline approximation which in general yields a good or nearly best spline approximation for free knots.

1. The Algorithm. In this section we describe the algorithm for computing a leveled set of knots $\left\{x_{1}, \ldots, x_{k}\right\}$.

We first state an obvious analogue of a well-known result on segment approximation given in Lawson [6] and Meinardus [7].

THEOREM 1.1. For a function d satisfying (0.1)-(0.3) the following statements hold:

(i) For every set of knots $a=x_{0} \leq x_{1} \leq \cdots \leq x_{k+1}=b$ we have

$$
\min _{0 \leq i \leq k} d\left(x_{i}, x_{i+1}\right) \leq m_{k} \leq \max _{0 \leq i \leq k} d\left(x_{i}, x_{i+1}\right) .
$$

(ii) Every leveled set of knots is optimal.

(iii) There exists a leveled set of knots.

(iv) If

$$
d(x, y)<d(\tilde{x}, \tilde{y}) \quad \text { for all }[x, y] \varsubsetneqq[\tilde{x}, \tilde{y}] \subset[a, b],
$$

then there exists a unique optimal set of knots. This set has $k$ distinct knots.

Description of the Algorithm. Let $d$ be a function satisfying (0.1)-(0.3). In the $n$th step $(n \geq 0)$ of the algorithm we will compute a set of knots $\left\{x_{1, n}, \ldots, x_{k, n}\right\}$ such that

$$
a=x_{0, n} \leq x_{1, n} \leq \cdots \leq x_{k, n} \leq x_{k+1, n}=b
$$

and we set

$$
d_{i, n}=d\left(x_{i, n}, x_{i+1, n}\right), \quad i=0, \ldots, k .
$$

To start the algorithm, we choose a set of knots $\left\{x_{1,0}, \ldots, x_{k, 0}\right\}$ (e.g., equidistant) such that

$$
\begin{gathered}
a=x_{0,0}<x_{1,0}<\cdots<x_{k, 0}<x_{k+1,0}=b, \\
d_{i, 0}>0, \quad i=0, \ldots, k,
\end{gathered}
$$


and compute these values. We set

$$
a_{0}=\min \left\{d_{i, 0}: i=0, \ldots, k\right\}
$$

and

$$
b_{0}=\max \left\{d_{i, 0}: i=0, \ldots, k\right\} .
$$

We proceed by induction as follows. For $n \geq 1$ we set

$$
d_{n}=\left(a_{n-1} b_{n-1}\right)^{1 / 2} \text {. }
$$

Then we compute a set of $\operatorname{knots}\left\{x_{1, n}, \ldots, x_{k, n}\right\}$ such that

$$
a=x_{0, n}<x_{1, n}<\cdots<x_{j_{n}, n}<x_{j_{n}+1, n}=\cdots=x_{k+1, n}=b
$$

and

$$
d_{i, n}=d_{n}, \quad i=0, \ldots, j_{n}-1 .
$$

(Note that $d_{j_{n}, n} \leq d_{n}$ if $j_{n}<k$.) We set $c_{n}=d_{k, n}$,

$$
a_{n}=\max \left\{a_{n-1}, \min \left\{c_{n}, d_{n}\right\}\right\} \quad \text { and } \quad b_{n}=\min \left\{b_{n-1}, \max \left\{c_{n}, d_{n}\right\}\right\} .
$$

In the $n$th step $(n \geq 1)$ we compute for $\mu=1, \ldots, j_{n}$ each knot $x_{\mu, n}$ by induction as follows: Having obtained

$$
a=x_{0, n}<x_{1, n}<\cdots<x_{i, n}
$$

for some index $i \geq 0$, we get the knot $x_{i+1, n}$ as the limit of a sequence $\left(\tilde{x}_{p}\right)$ which is defined as follows. Choose points $\tilde{x}_{1}$ and $\tilde{x}_{2}$ such that

$$
d\left(x_{i, n}, \tilde{x}_{1}\right) \leq d_{n}<d\left(x_{i, n}, \tilde{x}_{2}\right) .
$$

Then apply the regula falsi method for the exponents $\tilde{\delta}_{1}, \delta_{n}, \tilde{\delta}_{2}$ by setting

$$
\tilde{x}_{3}=\tilde{x}_{1}+\left(\tilde{x}_{2}-\tilde{x}_{1}\right)\left(\delta_{n}-\tilde{\delta}_{1}\right) /\left(\tilde{\delta}_{2}-\tilde{\delta}_{1}\right),
$$

where

$$
d_{n}=10^{\delta_{n}}, \quad d\left(x_{i, n}, \tilde{x}_{1}\right)=10^{\tilde{\delta}_{1}} \text { and } \quad d\left(x_{i, n}, \tilde{x}_{2}\right)=10^{\tilde{\delta}_{2}} .
$$

Then either

$$
d\left(x_{i, n}, \tilde{x}_{1}\right) \leq d_{n}<d\left(x_{i, n}, \tilde{x}_{3}\right)
$$

or

$$
d\left(x_{i, n}, \tilde{x}_{3}\right) \leq d_{n}<d\left(x_{i, n}, \tilde{x}_{2}\right) .
$$

In the first (respectively second) case, analogously as above we compute $\tilde{x}_{4}$ by regula falsi for the exponents $\tilde{\delta}_{1}, \delta_{n}, \tilde{\delta}_{3}$ (respectively $\tilde{\delta}_{3}, \delta_{n}, \tilde{\delta}_{2}$ ), where $d\left(x_{i, n}, \tilde{x}_{3}\right)=10^{\tilde{\delta}_{3}}$, and proceed by induction. (For another possibility of computing the set of knots $\left\{x_{1, n}, \ldots, x_{j_{n}, n}\right\}$ see $[10]$.)

The following convergence result can be proved analogously as Theorem 3.1 in [10].

THEOREM 1.2. If $m_{k}=10^{\delta}, d_{n}=10^{\delta_{n}}, a_{n}=10^{\alpha_{n}}$ and $b_{n}=10^{\beta_{n}}$ for all $n$, then

for all $n$ and

$$
\left|\delta-\delta_{n}\right| \leq \frac{1}{2}\left|\alpha_{n-1}-\beta_{n-1}\right| \leq \cdots \leq \frac{1}{2^{n}}\left|\alpha_{0}-\beta_{0}\right|
$$

$$
\lim _{n \rightarrow \infty} d_{n}=m_{k}
$$

In general, condition (1.2) is satisfied and therefore, since $\lim _{n \rightarrow \infty} d_{n}=m_{k}$, it is easily verified that the sequence $\left\{x_{1, n}, \ldots, x_{k, n}\right\}$ converges to the unique optimal set of knots $\left\{x_{1}, \ldots, x_{k}\right\}$. For a more general convergence result, which also holds for our algorithm, see [10]. 
2. Application to Piecewise Polynomials with Free Knots. In this section we describe a two-phase method for computing best piecewise polynomial approximations with free knots and introduce several functions $d$ which can be used in place of the minimal deviation in phase one of the method.

Let $C[a, b]$, the space of all continuous real-valued functions on an interval $[a, b]$, be endowed with the supremum norm

$$
\|f\|=\sup \{|f(t)|: t \in[a, b]\} .
$$

Moreover, for $r \geq 1$ we denote by $C^{r}[a, b]$ the subspace of functions in $C[a, b]$ which are $r$-times continuously differentiable.

The set of piecewise polynomials of degree $m \geq 1$ with $k \geq 1$ free knots is defined by

$$
\begin{aligned}
& P P_{m, k}=\{s:[a, b] \rightarrow \mathbf{R}: \text { there exist knots } \\
& \qquad \begin{array}{l}
a=x_{0}<x_{1}<\cdots<x_{k+1}=b \text { such that } \\
\left.\left.s\right|_{\left[x_{i}, x_{i+1}\right)} \in \Pi_{m}, i=0, \ldots, k-1, \text { and }\left.s\right|_{\left[x_{k}, x_{k+1}\right]} \in \Pi_{m}\right\} .
\end{array}
\end{aligned}
$$

A function $p_{f} \in P P_{m, k}$ is called a best approximation of a given function $f \in C[a, b]$, if

$$
\left\|f-p_{f}\right\|=\inf \left\{\|f-p\|: p \in P P_{m, k}\right\} .
$$

The problem of best approximation is equivalent to determining knots

$$
a=x_{0} \leq x_{1} \leq \cdots \leq x_{k} \leq x_{k+1}=b
$$

which satisfy $(0.5)$ for

$$
d\left(x_{i}, x_{i+1}\right)=d\left(f, \Pi_{m},\left[x_{i}, x_{i+1}\right]\right), \quad i=0, \ldots, k,
$$

where

$$
d\left(f, \Pi_{m},\left[x_{i}, x_{i+1}\right]\right)=\min _{p \in \Pi_{m}}\|f-p\|_{\left[x_{i}, x_{i+1}\right]}, \quad i=0, \ldots, k .
$$

To solve this problem, we compute a leveled set of knots which by Theorem 1.1 is optimal. This could be done by applying our algorithm directly to the function $d$ as in (2.1). Then in each step of the algorithm we have to compute minimal deviations as in (2.1) by using the classical Remez algorithm (see [7]).

In order to obtain a faster algorithm, in phase one of our method we replace the function in (2.1) by a suitable function $d$ which can be easily evaluated, and we compute by our algorithm a leveled set of knots for this function. (The choice of such type of suitable functions will be described subsequently.) Our numerical results show that by this approach, in most cases, we already obtain a nearly best piecewise polynomial (which sometimes may suffice for practical purposes).

Then in phase two we apply our algorithm to the function $d$ in (2.1) by using the knots computed in phase one as starting points.

In the following we describe various functions $d$ which can be used in phase one. In particular, these functions satisfy (0.1)-(0.3). The choice of $d$ depends on properties of the functions to be approximated and therefore we distinguish certain function classes.

2.1. Holomorphic Functions. Let $A[\alpha, \beta]$ be the space of all functions $f$ which are holomorphic in a region $G$ of the complex plane, where $[\alpha, \beta] \subset \operatorname{int} G$ and $f(t)$ 
is real for all $t \in[\alpha, \beta]$. If $f \in A[\alpha, \beta]$, we denote by $E_{r}$ an ellipse with foci $\alpha$ and $\beta$ such that $f$ is holomorphic in int $E_{r}$, where $r=p+q$ is the sum of the half axes $p$ and $q$ of $E_{r}$. Moreover, let $r(f)$ be the supremum of all such numbers $r$, with corresponding half axes $p(f)$ and $q(f)$. Then $E_{r(f)}$ is called the regularity ellipse of $f$.

We will use the following theorem of S. N. Bernstein (see [7]): For all $f \in$ $A[-1,1]$,

$$
\limsup _{m \rightarrow \infty} d\left(f, \Pi_{m},[-1,1]\right)^{1 / m}=1 /(p(f)+q(f)) .
$$

This result shows that we can take the value $(1 /(p(f)+q(f)))^{m}$ as an approximation of $d\left(f, \Pi_{m},[-1,1]\right)$.

Now, let a function $f \in A[a, b]$ be given which we want to approximate by $P P_{m, k}$. Moreover, assume that $z=(u, v) \in \partial E_{r(f)}$ such that $f$ is not holomorphic at $z$. For some partition $a=x_{0}<x_{1}<\cdots<x_{k}<x_{k+1}=b$ and $i \in\{0, \ldots, k\}$ we define $E_{i}$ as the ellipse with foci $x_{i}$ and $x_{i+1}$ such that $z \in \partial E_{i}$ and assume that $f$ is holomorphic on int $E_{i}, i=0, \ldots, k$. Let an integer $i \in\{0, \ldots, k\}$ be given. By applying a suitable linear transformation to $E_{i}$ we obtain an ellipse $\tilde{E}_{i}$ with foci -1 and 1. We now use the above result of Bernstein. Instead of computing directly a leveled set of knots for $d\left(f, \Pi_{m},\left[x_{i}, x_{i+1}\right]\right)$, we first compute in phase one of our method a leveled set of knots for the approximate value

$$
\tilde{d}\left(x_{i}, x_{i+1}\right)=\left(1 /\left(p_{i}(f)+q_{i}(f)\right)\right)^{m}
$$

where $p_{i}(f)$ and $q_{i}(f)$ are the half axes of $\tilde{E}_{i}$. Since the small half axis $q_{i}(f)$ is uniquely determined by the big half axis $p_{i}(f)$, it suffices to compute a leveled set of knots for

$$
d\left(x_{i}, x_{i+1}\right)=1 / p_{i}(f)
$$

where

$$
p_{i}(f)=\left[\left(\left(u-x_{i}\right)^{2}+v^{2}\right)^{1 / 2}+\left(\left(u-x_{i+1}\right)^{2}+v^{2}\right)^{1 / 2}\right] /\left(x_{i+1}-x_{i}\right) .
$$

2.2. Differentiable Functions. We use the following result of de Boor [1], [2] for differentiable functions: If $f \in C[\alpha, \beta] \cap C^{m+1}(\alpha, \beta]$ and $\left|f^{(m+1)}\right|$ is monotone decreasing on $[\alpha, \beta]$, then

$$
d\left(f, \Pi_{m},[\alpha, \beta]\right) \leq \frac{1}{(m+1) !}\left(\int_{\alpha}^{\beta}\left|f^{(m+1)}(t)\right|^{1 /(m+1)} d t\right)^{m+1}
$$

In view of this result we compute a leveled set of knots for

$$
d\left(x_{i}, x_{i+1}\right)=\int_{x_{i}}^{x_{i+1}}\left|f^{(m+1)}(t)\right|^{1 /(m+1)} d t, \quad i=0, \ldots, k .
$$

A further possibility is to use theorems of D. Jackson (see [11, p. 23]) and S. N. Bernstein (see [7, p. 78]) which can be summarized as follows: There exists a constant $K$ such that for every integer $j \in\{1, \ldots, m+1\}$ and every function $f \in C^{j}[\alpha, \beta]$

$$
d\left(f, \Pi_{m},[\alpha, \beta]\right) \leq K(\beta-\alpha)^{j}\left\|f^{(j)}\right\|_{[\alpha, \beta]} .
$$

In this case we compute a leveled set of knots for

$$
\left.d\left(x_{i}, x_{i+1}\right)=\left(x_{i+1}-x_{i}\right)^{j}\left\|f^{(j)}\right\|_{\left[x_{i}, x_{i}+1\right.}\right], \quad i=0, \ldots, k
$$

(where $j \in\{1, \ldots, m+1\}$ is independent of $i$ ). 
2.3. Continuous Functions. In this case we use a well-known lower bound for the minimal deviation.

Given a function $f \in C[\alpha, \beta]$, we define for all $i \in\{0, \ldots, m+1\}$

$$
t_{i}^{(m)}=\frac{\beta-\alpha}{2} \cos \frac{(m+1-i) \pi}{m+1}+\frac{\beta+\alpha}{2}
$$

and

$$
L_{m}(f,[\alpha, \beta])=f\left(t_{0}^{(m)}\right)+2 \sum_{i=1}^{m}(-1)^{i} f\left(t_{i}^{(m)}\right)+(-1)^{m+1} f\left(t_{m+1}^{(m)}\right) .
$$

Then we have

$$
\frac{1}{2 m+2}\left|L_{m}(f,[\alpha, \beta])\right| \leq d\left(f, \Pi_{m},[\alpha, \beta]\right)
$$

and

$$
L_{m}(p,[\alpha, \beta])=0 \quad \text { for all } p \in \Pi_{m} .
$$

The points defined in (2.5) are the extreme points of the Chebyshev polynomial of degree $m+1$ (see [7]).

We give an interpretation of the lower bound (2.7). If we choose in the first step of the classical Remez algorithm the points in (2.5), then we have to compute a polynomial $p \in \Pi_{m}$ and a real number $\lambda$ such that

$$
(-1)^{i}\left(f\left(t_{i}^{(m)}\right)-p\left(t_{i}^{(m)}\right)\right)=\lambda, \quad i=0, \ldots, m+1 .
$$

Then it can be shown that

$$
|\lambda|=\frac{1}{2 m+2}\left|L_{m}(f,[\alpha, \beta])\right| .
$$

In general, the polynomial $p$ is a good approximation for $f$ on $[\alpha, \beta]$ and the value $|\lambda|$ is a good approximation of $d\left(f, \Pi_{m},[\alpha, \beta]\right)$. (For details see [7].)

In view of these results we compute a leveled set of knots for the approximate value

$$
d\left(x_{i}, x_{i+1}\right)=\frac{1}{2 m+2}\left|L_{m}\left(f,\left[x_{i}, x_{i+1}\right]\right)\right|, \quad i=0, \ldots, k .
$$

In contrast to the functions $d$ in Subsections 2.1 and 2.2, the function $d$ in (2.9) does not satisfy (0.3), in general. However, in some cases our algorithm worked, although (0.3) was not satisfied (see, e.g., Example 1 in Section 3).

Moreover, we are able to prove that for a special class of functions, (2.9) satisfies (0.1)-(0.3).

THEOREM 2.1. For a function $f \in C[\alpha, \beta] \cap C^{m+1}(\alpha, \beta)$ the following statements are equivalent:

(i) The function $d: D=\left\{(x, y) \in \mathbf{R}^{2}: \alpha \leq x \leq y \leq \beta\right\} \rightarrow \mathbf{R}$, defined by

$$
d(x, y)=\frac{1}{2 m+2}\left|L_{m}(f,[x, y])\right|, \quad(x, y) \in D
$$

satisfies (0.1)-(0.3) and (1.2).

(ii) The function $f$ satisfies

$$
\sigma f^{(m+1)}(t) \geq 0, \quad t \in(\alpha, \beta), \sigma \in\{-1,1\},
$$

and $f^{(m+1)}$ does not vanish identically on a subinterval of $[\alpha, \beta]$. 
Proof. We first derive some formulae for $L_{m}(f,[x, y])$ and for the derivative of $L_{m}$ at $y$ (respectively at $x$ ), where $[x, y] \subset[\alpha, \beta)$ (respectively $[x, y] \subset(\alpha, \beta]$ ). Let an interval $[x, y] \subset[\alpha, \beta)$ be given. Let $p_{0} \in \Pi_{m}$ satisfy

$$
p_{0}\left(t_{i}^{(m)}\right)=f\left(t_{i}^{(m)}\right), \quad i=0, \ldots, m,
$$

where

$$
t_{i}^{(m)}=\frac{y-x}{2} \cos \frac{(m+1-i) \pi}{m+1}+\frac{y+x}{2}, \quad i=0, \ldots, m+1 .
$$

Now we use a well-known result on the error of interpolation for functions $f \in$ $C^{m+1}[\alpha, \beta]$ (see $[8$, p. 69]). It can be easily seen that the result is also true, if $f \in C[\alpha, \beta] \cap C^{m+1}(\alpha, \beta)$. Then we obtain

$$
\begin{aligned}
L_{m}(f,[x, y]) & =L_{m}(f,[x, y])-L_{m}\left(p_{0},[x, y]\right) \\
& =(-1)^{m+1}\left(f(y)-p_{0}(y)\right) \\
& =(-1)^{m+1}\left(y-t_{0}^{(m)}\right) \cdots \cdots\left(y-t_{m}^{(m)}\right) \frac{f^{(m+1)}\left(t_{0}\right)}{(m+1) !}
\end{aligned}
$$

for some $t_{0} \in(x, y)$.

Now let $x$ be fixed. Since, by (2.8), for all $p \in \Pi_{m}$

$$
L_{m}(p,[x, y])=0, \quad y \in(x, \beta),
$$

it follows that

$$
\frac{d}{d y} L_{m}(p,[x, y])=0, \quad y \in(x, \beta)
$$

Let $q_{1} \in \Pi_{m-1}$ satisfy

$$
q_{1}\left(t_{i}^{(m)}\right)=f^{\prime}\left(t_{i}^{(m)}\right), \quad i=1, \ldots, m,
$$

and let $p_{1} \in \Pi_{m}$ be such that $p_{1}^{\prime}=q_{1}$.

Analogously as above we obtain

$$
\begin{aligned}
\frac{d}{d y} L_{m}(f,[x, y]) & =\frac{d}{d y} L_{m}(f,[x, y])-\frac{d}{d y} L_{m}\left(p_{1},[x, y]\right) \\
& =(-1)^{m+1}\left(f^{\prime}(y)-q_{1}(y)\right) \\
& =(-1)^{m+1}\left(y-t_{1}^{(m)}\right) \cdots \cdot\left(y-t_{m}^{(m)}\right) \frac{f^{(m+1)}\left(t_{1}\right)}{m !}
\end{aligned}
$$

for some $t_{1} \in(x, y)$.

Repeated application of the above arguments yields

$$
\frac{d^{r}}{d y^{r}} L_{m}(f,[x, y])=(-1)^{m+1}\left(y-t_{r}^{(m)}\right) \cdots\left(y-t_{m}^{(m)}\right) \frac{f^{(m+1)}\left(t_{r}\right)}{(m+1-r) !}
$$

for some $t_{r} \in(x, y), r=0, \ldots, m$.

Analogous formulae hold for the derivatives of $L_{m}$ at $x$, where $[x, y] \subset(\alpha, \beta]$ and $y$ is fixed. We first show that (ii) implies (i). If $m=0$, then by hypothesis, $f$ is strictly monotone on $[\alpha, \beta]$ and statement (i) is easily verified. If $m=1$, then obviously

$$
\frac{d}{d y} L_{1}(f,[x, y])=-f^{\prime}\left(\frac{x+y}{2}\right)+f^{\prime}(y) \text { if } \alpha \leq x<y<\beta .
$$


Since by assumption $f^{\prime}$ is strictly monotone on $(\alpha, \beta)$, it follows that

$$
\frac{d}{d y} L_{1}(f,[x, y]) \neq 0 \text { if } \alpha \leq x<y<\beta
$$

Hence,

$$
d(x, y)<d(x, \tilde{y}) \quad \text { if } \alpha \leq x \leq y<\tilde{y}<\beta .
$$

Analogously, it follows that

$$
d(\tilde{x}, y)<d(x, y) \quad \text { if } \alpha<x<\tilde{x} \leq y \leq \beta .
$$

Then it is easily verified that $d$ satisfies statement (i). Assume, therefore, that $m \geq 2$. Without loss of generality we may assume that $m$ is odd and $f^{(m+1)}(t) \geq 0$ if $t \in(\alpha, \beta)$. Then (2.12) implies that

$$
\frac{d^{r}}{d y^{r}} L_{m}(f,[x, y]) \geq 0, \quad r=0, \ldots, m,
$$

for any interval $[x, y] \subset[\alpha, \beta)$. Therefore,

$$
d(x, y) \leq d(x, \tilde{y}) \quad \text { if } \alpha \leq x \leq y \leq \tilde{y}<\beta .
$$

Analogously, it follows that

$$
d(x, y) \leq d(\tilde{x}, y) \quad \text { if } \alpha<\tilde{x} \leq x \leq y \leq \beta .
$$

This implies that

$$
d(x, y) \leq d(\tilde{x}, \tilde{y}) \quad \text { if }[x, y] \subset[\tilde{x}, \tilde{y}] \subset[\alpha, \beta] .
$$

This proves (0.3). Obviously, $d$ also satisfies (0.1) and (0.2). Now we show that $d$ satisfies (1.2). Assume that

$$
d(\tilde{x}, \tilde{y})=d(\tilde{x}, \tilde{\tilde{y}}) \quad \text { for some } \alpha \leq \tilde{x} \leq \tilde{y}<\tilde{\tilde{y}}<\beta .
$$

Then by $(0.3)$,

$$
d(\tilde{x}, y)=d(\tilde{x}, \tilde{y}) \quad \text { if } y \in(\tilde{y}, \tilde{\tilde{y}})
$$

Therefore,

$$
\frac{d}{d y} L_{m}(f,[\tilde{x}, y])=0 \quad \text { if } y \in(\tilde{y}, \tilde{\tilde{y}})
$$

Since $f^{(m+1)}$ does not vanish identically on a subinterval of $(\alpha, \beta)$, there exists $\tilde{t} \in(\tilde{y}, \tilde{\tilde{y}})$ such that $f^{(m+1)}(\tilde{t})>0$, and therefore an interval $[u, v] \subset(\tilde{x}, \tilde{\tilde{y}})$ such that

$$
f^{(m+1)}(t)>0 \quad \text { if } t \in[u, v] .
$$

Then it follows from (2.11) that

$$
\left.\frac{d}{d y} L_{m}(f,[u, y])\right|_{y=v}>0 .
$$

Now (2.13) implies that

$$
\hat{d}(x, y)=\frac{d}{d y} L_{m}(f,[x, y]) \quad \text { if } \alpha \leq x \leq y<\beta
$$

satisfies (0.3). 
Then, by (2.14) and (2.15),

$$
0<\hat{d}(u, v) \leq \hat{d}(\tilde{x}, y)=0 \quad \text { if } y \in(\tilde{y}, \tilde{\tilde{y}}),
$$

a contradiction. This shows that

$$
d(x, y)<d(x, \tilde{y}) \quad \text { if } \alpha \leq x \leq y<\tilde{y}<\beta .
$$

Analogously, it follows that

$$
d(\tilde{x}, y)<d(x, y) \quad \text { if } \alpha<x<\tilde{x} \leq y \leq \beta .
$$

Then we obtain

$$
d(x, y)<d(\tilde{x}, \tilde{y}) \quad \text { if }[x, y] \varsubsetneqq[\tilde{x}, \tilde{y}] \subset[\alpha, \beta] .
$$

This proves that (ii) implies (i).

Now we show that (i) implies (ii). Since $d(x, x)=0$ and $d$ satisfies (1.2), it follows that

$$
d(x, y)>0 \quad \text { if } \alpha \leq x<y \leq \beta
$$

Hence,

$$
L_{m}(f,[x, y]) \neq 0 \quad \text { if } \alpha \leq x<y \leq \beta .
$$

Assume now that $f^{(m+1)}(t)=0$ if $t \in[x, y]$ for $\alpha<x<y<\beta$. Then by (2.10),

$$
L_{m}(f,[x, y])=0
$$

a contradiction to (2.16). Finally, assume that there exist $\tilde{t}, \tilde{\tilde{t}} \in(\alpha, \beta), \tilde{t}<\tilde{\tilde{t}}$, such that

$$
f^{(m+1)}(\tilde{t})<0, \quad f^{(m+1)}(\tilde{\tilde{t}})>0 .
$$

Then there exist intervals $[\tilde{x}, \tilde{y}] \subset(\alpha, \beta),[\tilde{\tilde{x}}, \tilde{\tilde{y}}] \subset(\alpha, \beta)$ with $\tilde{y}<\tilde{\tilde{x}}$ and

$$
\begin{array}{ll}
f^{(m+1)}(t)<0 & \text { if } t \in[\tilde{x}, \tilde{y}], \\
f^{(m+1)}(t)>0 & \text { if } t \in[\tilde{\tilde{x}}, \tilde{\tilde{y}}] .
\end{array}
$$

Then by $(2.10), L_{m}(f,[\tilde{x}, \tilde{y}])<0$ and $L_{m}(f,[\tilde{\tilde{x}}, \tilde{\tilde{y}}])>0$. Let $\tilde{x}$ be fixed. Since $L_{m}(f,[\tilde{\tilde{x}}, y])$ is continuous for $y \in[\tilde{y}, \tilde{\tilde{y}}]$, it follows that

$$
L_{m}(f,[\tilde{x}, \tilde{\tilde{y}}])<0,
$$

because otherwise there exists $\hat{y} \in(\tilde{y}, \tilde{\tilde{y}})$ such that $L_{m}(f,[\tilde{x}, \hat{y}])=0$, a contradiction to (2.16). Analogously, it can be shown that $L_{m}(f,[\tilde{x}, \tilde{\tilde{y}}])>0$, which contradicts (2.17). This completes the proof of the theorem.

We now consider approximation of functions $f \in C(T)$ by $P P_{m, k}$, where $T=$ $\left\{t_{1}, \ldots, t_{N}\right\}$ is a finite subset of $[a, b]$ consisting of "many" points. Such an approximation problem arises frequently in practice. It was noted in [10] that the algorithm for $C[a, b]$ can be applied to the above situation.

Now, analogously as in (2.4), by replacing the second derivative $f^{\prime \prime}$ by the second divided difference of $f$, one can use the function

$$
\begin{aligned}
d\left(x_{i}, x_{i+1}\right)=\left(x_{i+1}-x_{i}\right)^{2} \max \{\mid & f\left(t_{j-1}\right)-2 f\left(t_{j}\right)+f\left(t_{j+1}\right) \mid: \\
& \left.\left\{t_{j-1}, t_{j}, t_{j+1}\right\} \subset T \subset\left[x_{i}, x_{i+1}\right]\right\} .
\end{aligned}
$$

We finally describe a further approach for functions $f \in C[a, b]$. If we want to approximate $f$ by piecewise polynomials from $P P_{m, k}$ for $m>1$, then we can first 
compute a leveled set of knots for $P P_{1, k}$ and then apply our algorithm to $P P_{m, k}$ by using the knots for $P P_{1, k}$ as starting points.

2.4. Good Spline Approximations with Free Knots. We briefly discuss the computation of good or nearly best spline approximations with free knots in connection with the above algorithm for piecewise polynomials.

Best uniform approximation by splines with free knots is a nonlinear approximation problem. Therefore, if Newton-type methods are used, there arise two main difficulties. First, such algorithms only converge if one starts with nearly optimal knots. Therefore, since in general nearly optimal knots are not known, the convergence of Newton-type methods is not guaranteed. Secondly, even if the algorithms converge, in general they only yield local best approximations.

In view of these difficulties we use a different method which yields a good or nearly best global approximation and which converges if we start with arbitrary (e.g., equidistant) knots.

Let a function $f \in C[a, b]$ be given which we want to approximate by functions from $S_{m, k}$, the set of splines of degree $m$ with $k$ free knots. In step one of the method we approximate $f$ by $P P_{m, k}$ and compute a corresponding leveled set of knots $a=x_{0}<x_{1}<\cdots<x_{k+1}=b$. This leveled set of knots reflects the critical parts of $f$ in the following sense. In those parts, where the intervals $\left[x_{i}, x_{i+1}\right]$ are relatively large, $f$ can be approximated efficiently by $\Pi_{m}$. On the other hand, in those parts, where relatively small knot-intervals appear, the function behaves badly with respect to polynomial approximation. Therefore, since splines are piecewise polynomials satisfying certain differentiability properties at the knots, we take the above computed leveled set of knots $\left\{x_{1}, \ldots, x_{k}\right\}$ for $P P_{m, k}$ as fixed knots and compute a best uniform approximation of $f$ from $S_{m}\left(x_{1}, \ldots, x_{k}\right)$, the space of splines of degree $m$ with $k$ fixed knots, by applying the Remez-type algorithm in [9].

Our approach works for arbitrary continuous functions $f \in C[a, b]$. Moreover, if $f$ satisfies certain differentiability properties, then we can also take, instead of a leveled set of knots for $P P_{m, k}$, the knots from phase one of our algorithm as fixed knots, which are easier to compute.

Actually, in the special case of differentiable functions $f$, Dodson [5], de Boor [1], [2] and Burchard [4] suggested to take the leveled set of knots for the functions $d$ from Subsection 2.2 as fixed knots for spline approximations. In the above papers the computation of such a leveled set of knots was only solved approximately, since no general algorithm was available (see also [3, p. 180]).

Moreover, in our method we can control how efficient the resulting spline approximation is, since

$$
d\left(f, P P_{m, k}\right) \leq d\left(f, S_{m, k}\right) \leq d\left(f, S_{m}\left(x_{1}, \ldots, x_{k}\right)\right) .
$$

In general, $d\left(f, P P_{m, k}\right)$ is strictly smaller than $d\left(f, S_{m, k}\right)$, since no continuity is required for the functions from $P P_{m, k}$. Therefore, the relevant error

$$
\left|d\left(f, S_{m, k}\right)-d\left(f, S_{m}\left(x_{1}, \ldots, x_{k}\right)\right)\right|
$$

is strictly smaller than the value

$$
\left|d\left(f, P P_{m, k}\right)-d\left(f, S_{m}\left(x_{1}, \ldots, x_{k}\right)\right)\right|,
$$


which can be computed. Moreover, we have a further control, because we can also compare the value $d\left(f, S_{m}\left(x_{1}, \ldots, x_{k}\right)\right)$ with $d\left(f, S_{m}\left(y_{1}, \ldots, y_{k}\right)\right)$ for equidistant knots $y_{1}, \ldots, y_{k}$ in $[a, b]$.

Our numerical results show that in general we obtain a good or nearly best global spline approximation with respect to free knots.

3. Numerical Results. We will give some numerical examples on best piecewise polynomial approximations and on good spline approximations with free knots.

We first consider piecewise polynomials with free knots and compare the minimal deviations for equidistant knots, for knots computed in phase one of the algorithm and for optimal knots. In all subsequent tables the first row in each box gives the minimal deviation for equidistant knots. The second and third rows give minimal deviations corresponding to knot partitions computed in phase one by using various functions $d$ which will be specified in each example. The fourth row gives the minimal deviation for optimal knots.

Example 1. $f(t)=1 /\left(1+t^{2}\right),[a, b]=[-5,5]$.

2nd row: $d\left(x_{i}, x_{i+1}\right)=\left(x_{i+1}-x_{i}\right) /\left(\left(1+x_{i}^{2}\right)^{1 / 2}+\left(1+x_{i+1}^{2}\right)^{1 / 2}\right)$ according to (2.2).

3rd row: $d\left(x_{i}, x_{i+1}\right)$ according to (2.9). (Note that our algorithm works, although the function $d$ in (2.9) does not satisfy (0.3).)

\begin{tabular}{l|c|c|c}
\hline \multirow{2}{*}{$\begin{array}{l}\text { No. of } \\
\text { knots } k\end{array}$} & \multicolumn{3}{|c}{ Degree $m$} \\
\cline { 2 - 4 } & 3 & 5 & 7 \\
\hline \multirow{4}{*}{3} & $2.950 \mathrm{E}-2$ & $1.880 \mathrm{E}-3$ & $7.010 \mathrm{E}-4$ \\
& $8.484 \mathrm{E}-3$ & $6.627 \mathrm{E}-4$ & $1.742 \mathrm{E}-5$ \\
& $5.877 \mathrm{E}-3$ & $3.134 \mathrm{E}-4$ & $1.406 \mathrm{E}-5$ \\
& $5.861 \mathrm{E}-3$ & $3.126 \mathrm{E}-4$ & $1.256 \mathrm{E}-5$ \\
\hline \multirow{5}{*}{5} & $1.320 \mathrm{E}-2$ & $9.040 \mathrm{E}-4$ & $7.541 \mathrm{E}-5$ \\
& $8.300 \mathrm{E}-4$ & $7.530 \mathrm{E}-5$ & $3.103 \mathrm{E}-6$ \\
& $5.455 \mathrm{E}-4$ & $4.462 \mathrm{E}-5$ & $9.083 \mathrm{E}-7$ \\
& $4.518 \mathrm{E}-4$ & $4.426 \mathrm{E}-5$ & $8.537 \mathrm{E}-7$ \\
\hline
\end{tabular}

Example 2. $f(t)=1 / t^{2},[a, b]=[0.1,1]$.

2nd row: $d\left(x_{i}, x_{i+1}\right)=\left(x_{i+1}-x_{i}\right) /\left(x_{i}+x_{i+1}\right)$ according to (2.2).

3rd row: $d\left(x_{i}, x_{i+1}\right)$ according to (2.9).

\begin{tabular}{l|c|c|c|c}
\hline \multirow{2}{*}{$\begin{array}{l}\text { No. } \\
\text { of knots } k\end{array}$} & \multicolumn{4}{|c}{ Degree $m$} \\
\cline { 2 - 5 } & 3 & 5 & 7 & 9 \\
\hline & $2.43 \mathrm{E}+0$ & $2.73 \mathrm{E}-1$ & $2.85 \mathrm{E}-2$ & $2.84 \mathrm{E}-3$ \\
& $2.39 \mathrm{E}-1$ & $6.77 \mathrm{E}-3$ & $1.75 \mathrm{E}-4$ & $4.31 \mathrm{E}-6$ \\
3 & $5.48 \mathrm{E}-2$ & $1.45 \mathrm{E}-3$ & $3.66 \mathrm{E}-5$ & $8.79 \mathrm{E}-7$ \\
& $5.41 \mathrm{E}-2$ & $1.42 \mathrm{E}-3$ & $3.60 \mathrm{E}-5$ & $8.78 \mathrm{E}-7$ \\
\hline & $1.13 \mathrm{E}+0$ & $7.93 \mathrm{E}-2$ & $5.13 \mathrm{E}-3$ & $3.17 \mathrm{E}-4$ \\
& $5.85 \mathrm{E}-2$ & $7.50 \mathrm{E}-4$ & $8.79 \mathrm{E}-6$ & $9.79 \mathrm{E}-8$ \\
5 & $1.09 \mathrm{E}-2$ & $1.27 \mathrm{E}-4$ & $1.44 \mathrm{E}-6$ & $1.56 \mathrm{E}-8$ \\
& $1.06 \mathrm{E}-2$ & $1.26 \mathrm{E}-4$ & $1.44 \mathrm{E}-6$ & $1.56 \mathrm{E}-8$ \\
\hline
\end{tabular}


Example 3. $f(t)=t(\ln t)-t,[a, b]=[0,1]$.

2nd row: $d\left(x_{i}, x_{i+1}\right)=x_{i+1}^{1 /(m+1)}-x_{i}^{1 /(m+1)}$ according to (2.3).

3rd row: $d\left(x_{i}, x_{i+1}\right)$ according to $(2.9)$.

\begin{tabular}{l|c|c|c}
\hline \multirow{2}{*}{$\begin{array}{l}\text { No. } \\
\text { of knots } k\end{array}$} & \multicolumn{3}{|c}{ Degree $m$} \\
\cline { 2 - 4 } & 3 & 5 & 7 \\
\hline & $6.088 \mathrm{E}-3$ & $2.239 \mathrm{E}-3$ & $1.150 \mathrm{E}-3$ \\
& $6.514 \mathrm{E}-4$ & $1.562 \mathrm{E}-4$ & $8.374 \mathrm{E}-5$ \\
3 & $4.227 \mathrm{E}-4$ & $5.049 \mathrm{E}-5$ & $1.052 \mathrm{E}-5$ \\
& $3.485 \mathrm{E}-4$ & $3.720 \mathrm{E}-5$ & $7.229 \mathrm{E}-6$ \\
\hline & $4.058 \mathrm{E}-3$ & $1.493 \mathrm{E}-3$ & $7.668 \mathrm{E}-4$ \\
& $1.250 \mathrm{E}-4$ & $1.454 \mathrm{E}-5$ & $4.248 \mathrm{E}-6$ \\
5 & $1.047 \mathrm{E}-4$ & $7.479 \mathrm{E}-6$ & $1.030 \mathrm{E}-6$ \\
& $8.276 \mathrm{E}-5$ & $5.183 \mathrm{E}-6$ & $6.440 \mathrm{E}-7$ \\
\hline
\end{tabular}

Example 4. $f(t)=t^{1 / 2},[a, b]=[0,1]$.

2nd row: $d\left(x_{i}, x_{i+1}\right)=x_{i+1}^{1 /(2 m+2)}-x_{i}^{1 /(2 m+2)}$ according to (2.3).

3rd row: $d\left(x_{i}, x_{i+1}\right)$ according to $(2.9)$.

\begin{tabular}{l|c|c|c}
\hline \multirow{2}{*}{$\begin{array}{l}\text { No. of } \\
\text { knots } k\end{array}$} & \multicolumn{3}{|c}{ Degree $m$} \\
\cline { 2 - 4 } & 3 & 5 & 7 \\
\hline \multirow{4}{*}{3} & $2.300 \mathrm{E}-2$ & $1.390 \mathrm{E}-2$ & $9.990 \mathrm{E}-3$ \\
& $3.799 \mathrm{E}-3$ & $3.270 \mathrm{E}-3$ & $3.468 \mathrm{E}-3$ \\
& $1.870 \mathrm{E}-3$ & $5.668 \mathrm{E}-4$ & $2.443 \mathrm{E}-4$ \\
& $1.397 \mathrm{E}-3$ & $3.991 \mathrm{E}-4$ & $1.568 \mathrm{E}-4$ \\
\hline & $1.875 \mathrm{E}-2$ & $1.138 \mathrm{E}-2$ & $8.158 \mathrm{E}-3$ \\
5 & $8.575 \mathrm{E}-4$ & $4.214 \mathrm{E}-4$ & $4.059 \mathrm{E}-4$ \\
& $5.735 \mathrm{E}-4$ & $1.211 \mathrm{E}-4$ & $3.960 \mathrm{E}-5$ \\
& $3.986 \mathrm{E}-4$ & $7.479 \mathrm{E}-5$ & $2.228 \mathrm{E}-5$ \\
\hline
\end{tabular}

We finally give some examples on good spline approximations with free knots. In the following tables the first row is $d\left(f, S_{m}\left(y_{1}, \ldots, y_{k}\right)\right)$, where $\left\{y_{1}, \ldots, y_{k}\right\}$ is the set of equidistant knots; the second row is $d\left(f, S_{m}\left(x_{1}, \ldots, x_{k}\right)\right)$, where $\left\{x_{1}, \ldots, x_{k}\right\}$ is the leveled set of knots for $P P_{m, k}$; and the third row is $d\left(f, P P_{m, k}\right)$. In all examples we consider the case when $m=3$ and $k=5$.

\begin{tabular}{l|l|l|l}
\hline$f(t)=1 /\left(1+t^{2}\right)$ & $f(t)=1 / t^{2}$ & $f(t)=t(\ln t)-t$ & $f(t)=t^{1 / 2}$ \\
{$[a, b]=[-5,5]$} & {$[a, b]=[0.1,1]$} & {$[a, b]=[0,1]$} & {$[a, b]=[0,1]$} \\
\hline $5.971 \mathrm{E}-2$ & $2.027 \mathrm{E}+0$ & $5.610 \mathrm{E}-3$ & $2.230 \mathrm{E}-2$ \\
$2.585 \mathrm{E}-3$ & $4.202 \mathrm{E}-2$ & $3.017 \mathrm{E}-4$ & $1.252 \mathrm{E}-3$ \\
$4.518 \mathrm{E}-4$ & $1.060 \mathrm{E}-2$ & $8.276 \mathrm{E}-5$ & $3.986 \mathrm{E}-4$ \\
\hline
\end{tabular}


Fakultät für Mathematik und Informatik

Universität Mannheim

D-6800 Mannheim, West Germany

Mathematisches Institut

Universität Erlangen-Nürnberg

D-8520 Erlangen, West Germany

Mathematisch-Geographische Fakultät

Katholische Universität Eichstätt

D-8078 Eichstätt, West Germany

Institut für Angewandte Mathematik

Universität Erlangen-Nürnberg

D-8520 Erlangen, West Germany

1. C. DE BOOR, "Good approximation by splines with variable knots," in Spline Functions and Approximation Theory (A. Meir and A. Sharma, eds.), Birkhäuser-Verlag, Basel, 1973, pp. 57-72.

2. C. DE BOOR, "Good approximation by splines with variable knots II," in Numerical Solution of Differential Equations (G. A. Watson, ed.), Lecture Notes in Math., vol. 363, Springer-Verlag, Berlin and New York, 1974, pp. 12-20.

3. C. DE BOoR, A Practical Guide to Splines, Springer-Verlag, Berlin and New York, 1978.

4. H. G. BuRChARD, "Splines (with optimal knots) are better," Applicable Anal., v. 3, 1974, pp. 309-319.

5. D. S. Dodson, Optimal Order Approximation by Polynomial Spline Functions, Ph. D. Thesis, Purdue University, West Lafayette, IN, 1972.

6. C. L. LAWSON, "Characteristic properties of the segmented rational minimax approximation problem," Numer. Math., v. 6, 1964, pp. 293-301.

7. G. MEINARDUS, Approximation of Functions: Theory and Numerical Methods, Springer-Verlag, Berlin and New York, 1967.

8. G. Meinardus \& G. Merz, Praktische Mathematik I, B. I.-Verlag, Mannheim, 1979.

9. G. NÜRNBERGER \& M. SOMMER, "A Remez type algorithm for spline functions," Numer. Math., v. 41, 1983, pp. 117-146.

10. G. NÜRNBERGER, M. SOMMER \& H. STRAUSS, "An algorithm for segment approximation," Numer. Math., v. 48, 1986, pp. 463-477.

11. T. J. RIVLIN, An Introduction to the Approximation of Functions, Blaisdell, Waltham, Mass., 1969. 